\title{
Reducing everyday memory and planning problems by means of a paging system: a randomised control crossover study
}

\author{
B A Wilson, H C Emslie, K Quirk, J J Evans
}

\begin{abstract}
Objectives-To evaluate a paging system designed to improve independence in people with memory problems and executive deficits.

Methods-After a successful pilot study, a randomised control trial was conducted involving a crossover design with 143 people aged between 8 and 83 years. All had one or more of the following: memory, planning, attention, or organisation problems. Most had sustained a traumatic head injury or a stroke although a few had developmental learning difficulties or other conditions. The crossover design ensured that some people received a pager after a 2 week baseline whereas others were required to wait for 7 weeks after the baseline before receiving the pager. Participants were assessed at three time periods-namely, at baseline, 7 weeks, and at 14 weeks postbaseline.

Results-More than $80 \%$ of those who completed the 16 week trial were significantly more successful in carrying out everyday activities (such as self care, self medication, and keeping appointments) when using the pager in comparison with the baseline period. For most of these, significant improvement was maintained when they were monitored 7 weeks after returning the pager.
\end{abstract}

Conclusions-This particular paging system significantly reduces everyday failures of memory and planning in people with brain injury.

(F Neurol Neurosurg Psychiatry 2001;70:477-482)

Keywords: memory; rehabilitation; brain injury

Although external aids are almost certainly the best compensatory strategies for people with impaired memory, ${ }^{1}$ it is true to say that such aids are difficult to use for many people with brain injury who have memory, planning, and organisational problems. The employment of external memory aids is in itself a memory task, so the people who need them most typically have the greatest problems in using them. People with cognitive difficulties often forget to record information and forget to access information that is already recorded. They have great difficulty in programming electronic aids and even simpler paper and pencil aids may be used in an unsystematic or disorganised way. Finally, external aids are embarrassing for some people with impaired memory.
To overcome these problems, Hersh and Treadgold ${ }^{2}$ devised a radio paging system, utilising an alphanumeric pager. The system involves an arrangement of microcomputers linked to a conventional computer memory and, by modem, to a paging company. Reminders for each individual using the system are entered into the computer and, at the appropriate date and time, the reminders are transmitted to the individual pager. An audio or vibration alarm alerts the wearer to the message on the pager that contains a reminder of the task to be carried out. The pager is clipped on a waistband or carried in a pocket. It is simple to operate even for those with motor difficulties.

We began evaluating this system in 1994 and published the results of our pilot study with 15 people with impaired memory in $1997 .{ }^{3}$ Every one of the 15 clients showed a statistically significant improvement in achieving everyday targets between the baseline period and when using this paging system. For the group as a whole the average success rate in the $4-6$ week baseline period was $37 \%$. This rose to $86 \%$ in the 12 week treatment (pager) phase and remained fairly high $(76 \%)$ in the posttreatment (2-4 weeks) phase. For some participants this represented virtually no decline after the pager had been returned, which suggests that the paging system might be very useful as a short term measure with people with impaired memory. This was demonstrated in a follow up study of one of these when the system was found useful in helping to establish routines and aid the transitions to independent living. ${ }^{4}$

However, the findings that a few participants returned to or around baseline level after returning the pager suggests that for some people provision of the paging system would have to be on a long term basis. One patient, a woman with marked problems in planning, organisation, and attention, was given an extended trial and was followed up at regular intervals. ${ }^{5}$ After 2 years she continued to need the pager. Such findings suggest that the presence or absence of executive impairments might determine whether provision of the paging system would be on a long term or short term basis.

In the present study we wanted to extend the findings of the original pilot study and also to use a randomised control design to further strengthen the suggestion that this paging system was indeed effective for reducing the everyday problems of people with brain injury. 


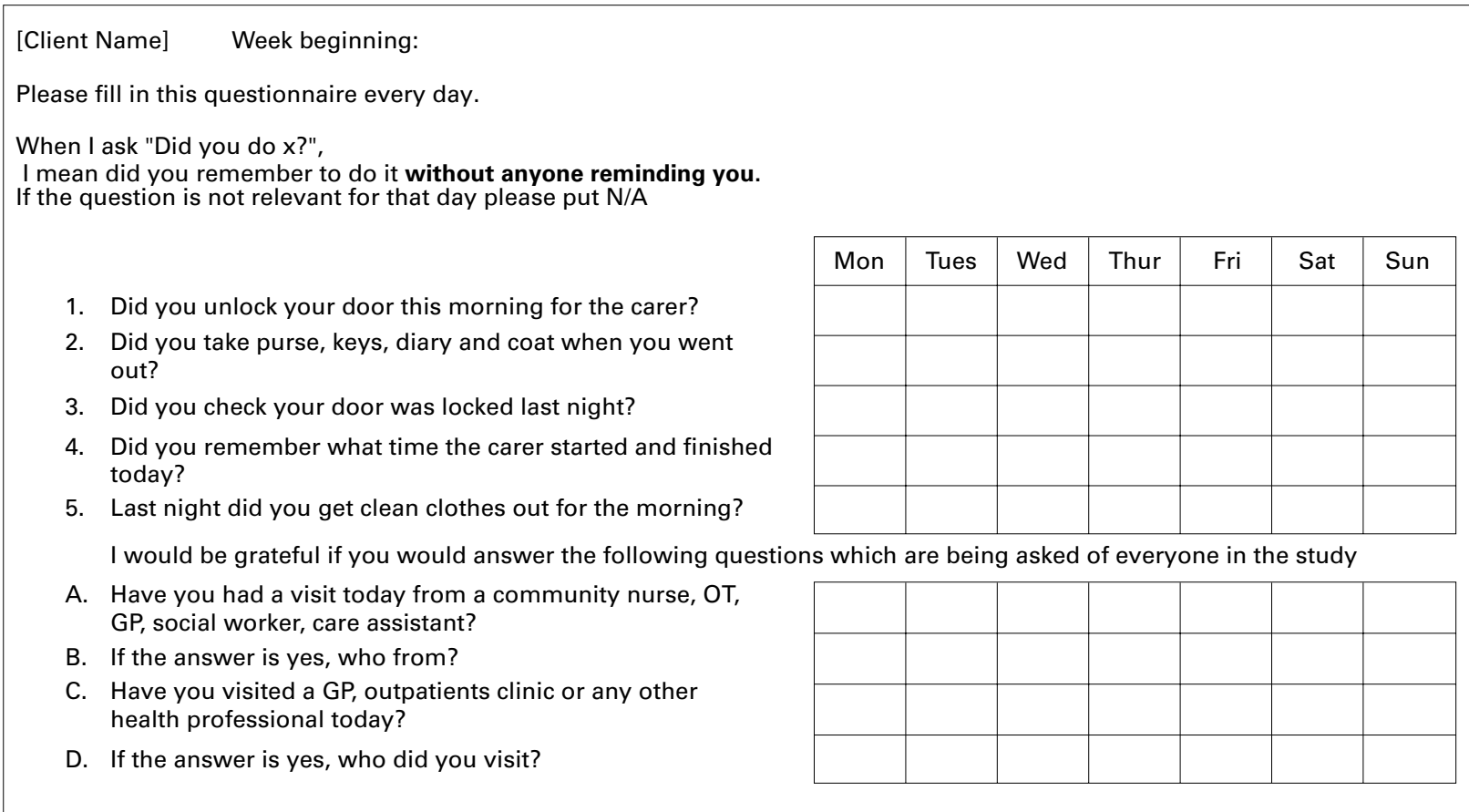

Figure 1 Example of a questionnaire.

We used a crossover design to avoid disappointment in those people not immediately allocated to a pager. We wanted to know whether or not the paging system enabled people with memory and planning problems after brain injury to carry out everyday tasks. Given the apparent robustness of the system, as indicated by the pilot study, we accepted a wide range of referrals and did not restrict ourselves to a highly selected group. We could then consider the question of whether the effectiveness is related to the kind of impairment (for example, memory or executive impairments), the degree of impairment (for example, mild, moderate, or severe) or both. Furthermore, in keeping with our belief that rehabilitation should target individual problems and enable people with brain injury to survive in their own, most appropriate environments, participants selected their own prompts that addressed their needs. They were also involved in establishing target behaviours to be monitored throughout the trial.

\section{Method}

DESIGN

A randomised control, crossover design was employed. After an initial assessment, target behaviours were measured for 2 weeks to establish a baseline (time 1). Participants were then randomly allocated to the treatment (pager) phase (group A) or to the waiting list (group B) for 7 weeks. Measures were taken during the last 2 weeks of this period (time 2). Conditions were then switched. Those given a pager were asked to return them and those on the waiting list were now given a pager. This stage also lasted 7 weeks and measures were taken during the last 2 weeks of this stage (time 3).

\section{PROCEDURE}

Potential participants were sent a letter explaining in some detail what would be involved in the trial. Those interested in taking part were seen for a 2-3 hour interview and assessment. As far as possible each participant was seen with a relative or carer. In this period, the pager was demonstrated and a trial message sent to ensure that the client could (a) read the message and (b) respond appropriately-that is, press the right button. People with memory or planning problems and their relatives or carers were then asked to go through a typical day to elicit problems that they might need help with. For example, if the person did not know what day it was on waking, it was suggested that a message could be sent saying "Good morning, $\mathrm{X}$, it is 730 am on Monday November $21 \mathrm{st}$ ". Only messages requested or agreed by the participant were selected for transmission. Participants also chose the wording of the messages and were free to modify these as necessary during the trial.

In addition to selecting the messages, each participant was given the following neuropsychological tests: (1) speed and capacity of language processing ${ }^{6}$ to estimate IQ and speed of information processing, (2) the Rivermead behavioural memory test ${ }^{7}$ to assess current memory functioning, (3) the map search test from the test of everyday attention ${ }^{8}$ to assess attention, and (4) the modified six elements test from the behavioural assessment of the dysexecutive syndrome ${ }^{9}$ to assess planning and organisational skills. These tests were only administered if they had not been administered by the referring clinicians within the past 6 months.

At the end of this consultation, participants were randomly assigned (with certain restrictions) to group A (to receive the pager after a 2 
Table 1 Characteristics of the participants

\begin{tabular}{|c|c|c|}
\hline & 1 (198 referrals) & $\begin{array}{l}2 \text { (143 participants who } \\
\text { completed all stages) }\end{array}$ \\
\hline Mean age (SD) (y) & $38.41(15.12)$ & $38.57(14.87)$ \\
\hline Age range & $8-83$ & $8-83$ \\
\hline Male/Female & $142: 56$ & $105: 38$ \\
\hline Mean age (SD) at time of insult & $33.45(15.94)$ & $33.25(16.25)$ \\
\hline Mean time (SD) (y) since insult & $5.16(5.92)$ & $4.9(6.25)$ \\
\hline Not in paid employment $(\%)$ & 92.4 & 93.7 \\
\hline Traumatic head injury (\%) & 43.4 & 44.1 \\
\hline Stroke $(\%)$ & 23.2 & 25.2 \\
\hline Acquired non-progressive brain injury (eg anoxia, meningitis, encephalitis) (\%) & 17.2 & 14.6 \\
\hline Acquired progressive brain injury (eg Alzheimer's, multiple sclerosis) (\%) & 7.6 & 7.0 \\
\hline Other conditions (eg developmental, diagnosis uncertain) (\%) & 8.6 & 9.1 \\
\hline Living in residential care/hospital (\%) & 4.6 & 4.2 \\
\hline Living with family (spouse/parent) (\%) & 68.2 & 72.0 \\
\hline Living alone with help of carer(s) (\%) & 13.1 & 12.6 \\
\hline Living alone (\%) & 14.1 & 11.2 \\
\hline
\end{tabular}

week baseline) or group B (to wait for 7 weeks after baseline before receiving the pager). The restrictions to the randomisation procedure were as follows:

(1) For five participants the 7 week pager phase would have included periods when the probable prompts/monitored target behaviours would be irrelevant (for example, reminders to pick the children up from school would not be relevant during the school holidays), thus these participants were assigned to the group where the pager phase did not coincide with school holidays. A similar approach was taken to holidays abroad, periods of respite care, etc. Three participants were assigned non-randomly for these reasons; two of these failed to complete the trials.

(2) Some inpatients: in a few cases referrers wanted to test the efficacy of the paging system in hospitals or rehabilitation units or wanted to see whether this could aid transition from hospital to home. If the referrers were certain that the patients would not still be inpatients in 3 or 4 months time (which was how long it would be before patients in group $B$ reached the pager phase), they were assigned to group A. Four patients were assigned non-randomly for these reasons. One later withdrew.

Finally, at the end of this initial consultation period, information was collected on the cause of brain injury, the time of the insult, current employment, and living situation.

A four to seven item questionnaire was devised for each participant based on the earlier discussions of current everyday memory and attention and other cognitive failures and possible reminders likely to be relevant throughout the 16 week study period. An example of a questionnaire is given in fig 1 .

Two copies of the questionnaire, together with a copy of the modified caregiver strain index $\left(\mathrm{MCGSI}^{10}\right.$ and Yool et al, unpublished data) were sent to each participant and carer in the week before the start of the baseline together with the agreed list of prompts and a stamped addressed envelope. The MCGSI, questionnaires, and envelopes were sent again just before the measures were due to be collected for times 2 and 3. Each participant also received a telephone call on the 1 st or 2 nd day after starting to use the pager to check that everything was going well. Participants or carers were asked to record daily whether or not targets had been achieved. They were also encouraged to add or delete non-target messages throughout the trial.

REFERRALS

Referrals were accepted from throughout the United Kingdom. Most were from clinical psychologists and occupational therapists, with others from speech and language therapists, psychiatrists, and organisations such as Headway (the National Head Injuries Association) and disability services.

We accepted anyone who was thought to have memory and/or planning/organisational difficulties, whether these were developmental in origin or acquired later in life. We set no upper or lower age limits provided that the person could read and was able to keep records (or have a carer willing to keep records). We also requested that participants should have some degree of insight in so far as acknowledging that they had some everyday memory, planning, or organisational problems and were willing to try to overcome these with the paging system.

RESPONSE RATE

Of 209 people referred for the trial, 11 withdrew (five before the first appointment, one died before the appointment, and two could not get funding for travel from their respective health authorities before the end of the trial).

Of the 198 clients who attended the first appointment, 18 did not proceed to the trial phase. For eight of these this was for reasons beyond their control (for example, poor pager reception in their home areas, transfers to other hospitals, and needing the pager for a job that did not materialise). A further six denied that they had problems, three had compensatory systems they were unwilling to abandon for the pager, and one was unable to read although this had not been apparent on the referrer's initial assessment.

Of the remaining 180 clients who began the baseline phase, seven (five of them teenagers) failed to return forms at the end of the baseline period and thus proceeded no further. In total, 173 clients completed the baseline period but one further client withdrew as she was completing her target behaviours successfully and, therefore, had no need of the pager.

A further 17 clients withdrew before the end of the trial, one because his vision was so poor 
Table 2 Demographic differences between group $A$ (pager first) and group $B$ (pager second)

\begin{tabular}{lll}
\hline Characteristic & Group A & Group B \\
\hline Mean current age (SD) (y) & $39.18(14.57)$ & $37.41(15.52)$ \\
Range & $10-83$ & $8-68$ \\
Mean age (SD) at insult (y) & $34.18(16.04)$ & $34.0(17.78)$ \\
Range & $0.5-80$ & $0-65$ \\
Mean time (SD) since insult (y) & $5.33(5.8)$ & $4.8(6.94)$ \\
Range & $0.5-26$ & $0.5-32$ \\
Male/female ratio & $70: 24$ & 35.14 \\
Traumatic head injury (\%) & 46.8 & 38.8 \\
Stroke (\%) & 25.5 & 24.5 \\
Acquired non-progressive conditions (\%) & 12.8 & 18.4 \\
With other conditions (\%) & 9.6 & 2.0 \\
Living in residential care/hospital (\%) & 4.3 & 4.1 \\
Living with family (\%) & 68.1 & 79.6 \\
Living alone with help of carer(s) (\%) & 13.8 & 10.2 \\
Living alone (\%) & 13.8 & 6.1 \\
Mean number of messages (SD) sent daily during & $8.78(4.05)$ & $8.26(3.96)$ \\
pager phase & & $2-25$ \\
Range & $4-25$ & $2-23$ \\
\hline
\end{tabular}

that it was too effortful to read the screen. One client lost the pager. Two were admitted to hospitals for treatment of cancer. The remaining clients no longer thought that the prompts from the pager were appropriate because their personal circumstances had changed.

Thus 156 clients completed all stages of the trial although 11 did not return the final set of forms and the forms of two clients were lost in the post leaving 143 clients with complete data. This 143 represents a response rate of $67.5 \%$ of the original $209,72 \%$ of the 198 who attended the first appointment and $79.4 \%$ of the 180 who proceeded to the baseline stage.

CHARACTERISTICS OF PARTICIPANTS

These are given in table 1 .

STATISTICAL METHODS

An odds ratio test ${ }^{11}$ was used to examine the differences in achievement of target behaviours during the three time periods in which they were monitored. This test takes into account different underlying success rates for each target and calculates an average improvement factor.

In addition we used $\chi^{2}$ tests to determine differences between total proportions of successes in group A and group B. We looked at targets achieved and not achieved for each group. We did this for each of the three time periods.

Results

DEMOGRAPHIC CHARACTERISTICS BETWEEN GROUPS A AND B

There were no significant differences between groups $\mathrm{A}$ and $\mathrm{B}$ in terms of current age or age at insult ( $\mathrm{p}=0.50$ and 0.58 respectively, $t$ tests), number of years after insult $(p=0.75$, Mann-
Whitney), sex ( $\mathrm{p}=0.70, \chi^{2}$, using groups $\mathrm{A}$ and $\mathrm{B}$ together with male or female), diagnostic group or independence category $(\mathrm{p}=0.10$ and 0.49 respectively, Fisher's exact test). There were no significant differences between the groups on any of the neuropsychological test results. The demographic and neuropsychological results are given in tables 2 and 3 .

The mean number of messages sent each day during the pager phase was 8.78 (SD 4.05) for group A and 8.26 (SD 3.96) for group B.

TARGETS ACHIEVED IN THE BASELINE PERIOD A $\chi^{2}$ test showed no significant difference in the proportion of targets hit and not hit between the two groups in the baseline period $(p=0.68)$. Group A achieved $46.82 \%$ of their targets and group B $48.63 \%$.

TARGETS ACHIEVED AT TIME 2

At time 2, group A participants were in the last 2 weeks of the 7 week period with the pager and group B were in the last 2 weeks of the waiting list period.

A $\chi^{2}$ test showed that group A was significantly more successful in achieving target behaviours than group B $(p<0.001)$. The percentage of targets achieved by group $A$ was $74.47 \%$ and by group B $48.18 \%$.

TARGETS ACHIEVED AT TIME 3

At time 3, group A participants no longer had pagers and group B were in the last 2 weeks of the pager phase.

A $\chi^{2}$ test showed that at this stage group B was more successful than group $A$ at achieving targets $(p<0.001)$. The percentage achieved by group A was $62.15 \%$ and by group B $76.13 \%$.

COMPARISON OF GROUP A PARTICIPANTS IN TIMES 1,2 , AND 3

There were significant differences between time periods for group A: time 1 versus time 2 $(p<0.001)$; time 2 versus time $3(p<0.001)$, and time 1 versus time $3(\mathrm{p}<0.001)$.

\section{COMPARISON OF GROUP B PARTICIPANTS IN TIMES} 1,2 , AND 3

There were no significant differences in the targets achieved by group B in times 1 and 2 - that is, baseline phase and waiting list phase $(p=0.75)$. There was a significant difference between time 1 (baseline) and time 3 (pager) $(p<0.001)$ and a significant difference between time 2 (waiting list) and time 3 (pager) $(\mathrm{p}<0.001)$

Table 3 Neuropsychological results for groups $A$ and $B$

\begin{tabular}{|c|c|c|c|c|c|c|c|c|c|c|c|c|c|c|}
\hline & \multicolumn{4}{|c|}{ Test of everyday attention } & \multicolumn{4}{|c|}{$R B M T$} & \multicolumn{4}{|c|}{ SCOLP } & \multirow{2}{*}{\multicolumn{2}{|c|}{$\begin{array}{l}\text { BADS } \\
\begin{array}{l}\text { Modified six } \\
\text { elements }\end{array}\end{array}$}} \\
\hline & \multicolumn{2}{|c|}{$\begin{array}{l}\text { Map search } 1 \\
\text { minute }\end{array}$} & \multicolumn{2}{|c|}{$\begin{array}{l}\text { Map search } 2 \\
\text { minutes }\end{array}$} & \multicolumn{2}{|c|}{ Profile score } & \multicolumn{2}{|c|}{ Screening score } & \multicolumn{2}{|c|}{$\begin{array}{l}\text { Semantic } \\
\text { processing }\end{array}$} & \multicolumn{2}{|c|}{ Spot the word } & & \\
\hline & $A$ & $B$ & $A$ & $B$ & $A$ & $B$ & $A$ & $B$ & $A$ & $B$ & $A$ & $B$ & $A$ & $B$ \\
\hline \multicolumn{15}{|c|}{ Scaled scores: } \\
\hline Mean & 5.65 & 6.18 & 4.83 & 5.65 & 11.22 & 12.64 & 4.29 & 4.96 & 6.3 & 6.91 & 8.7 & 9.1 & 2.29 & 2.28 \\
\hline SD & 3.76 & 3.43 & 3.8 & 3.56 & 6.7 & 6.35 & 3.36 & 3.17 & 3.3 & 3.52 & 3.2 & 3.17 & 1.35 & 1.43 \\
\hline Range & $0-14$ & $0-13$ & $0-18$ & $0-14$ & $0-23$ & $0-23$ & $0-11$ & $0-11$ & $0-17$ & $0-17$ & $0-16$ & $2-15$ & $0-4$ & $0-4$ \\
\hline & \multicolumn{2}{|c|}{$\begin{array}{l}t(130)=-0.82 \\
\mathrm{p}=0.42\end{array}$} & \multicolumn{2}{|c|}{$\begin{array}{l}t(130)=1.23 \\
\mathrm{p}=0.22\end{array}$} & \multicolumn{2}{|c|}{$\begin{array}{l}t(130)=1.20 \\
\mathrm{p}=0.24\end{array}$} & \multicolumn{2}{|c|}{$\begin{array}{l}\mathrm{t}(130)=1.18 \\
\mathrm{p}=0.24\end{array}$} & \multicolumn{2}{|c|}{$\begin{array}{l}t(130)=0.96 \\
\mathrm{p}=0.34\end{array}$} & \multicolumn{2}{|c|}{$\begin{array}{l}t(130)=0.69 \\
\mathrm{p}=0.50\end{array}$} & \multicolumn{2}{|c|}{$\begin{array}{l}t(130)=-0.04 \\
\mathrm{p}=0.96\end{array}$} \\
\hline
\end{tabular}

SCOLP $=$ Speed and capacity of language processing; RBMT $=$ Rivermead behavioural memory test. 


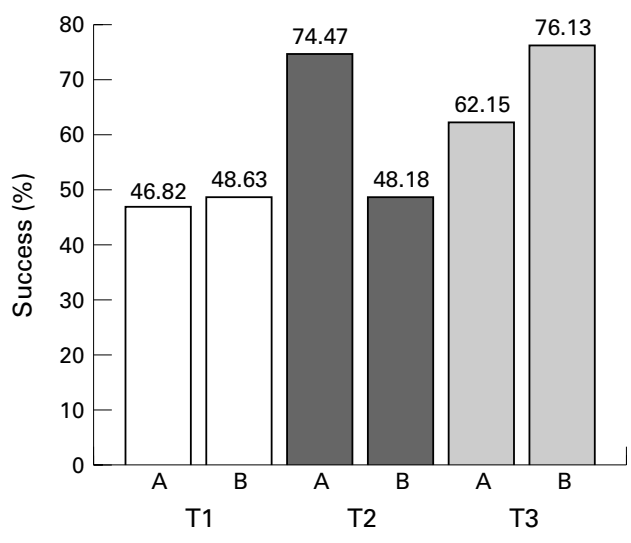

Figure 2 Percentage success rate for participants in group $A$ (pager first) and group $B$ (pager later) at time 1 (T1; baseline); time 2 (T2; weeks 8 and 9), and time 3 (T3; weeks 15 and 16).

The main results are shown in figure 2 .

COMPARISON OF GROUPS A AND B BETWEEN BASELINE AND PAGER PHASES: TIME 1 VERSUS TIME 2 FOR GROUP A AND TIME 1 VERSUS TIME 3 FOR GROUP B

Using the odds ratio test, of the 143 participants completing all phases, 121 (84.6\%) were significantly more successful with the pager than they had been in the baseline phase. A further seven people (4.9\%) showed improvement between the two phases (although this was not significant), nine (6.3\%) showed no real difference, and six (4.2\%) were actually worse with the pager than in the baseline phase.

COMPARISON OF GROUP A SUCCESSFUL USERS BETWEEN PAGER AND POSTPAGER PHASES: TIME 2 VERSUS TIME 3

Postpager data were available only for group A. Of the 74 participants in this group who were significantly more successful during the pager phase than the baseline phase, 54 (73\%) were still significantly better than at baseline, 7 weeks after returning their pager.

RELATION TO DEMOGRAPHIC VARIABLES

A multiple regression equation was used to see whether the following variables were related to successful use of the pager (as measured by the odds ratio test): current age, age at insult, time since insult, sex, diagnostic group, number of messages sent a day, level of independent living (living alone, living with carers-family or professional, living with spouse, or living in a hospital/rehabilitation unit) and neuropsychological test scores. Complete data were available for 136 of the 143 participants.

The only variables that were related to treatment outcome were $\operatorname{sex}\left(\mathrm{F}_{1,119}=5.69, \mathrm{p}=0.02\right)$ and diagnostic group $\left(\mathrm{F}_{4,119}=2.85, \mathrm{p}=0.03\right)$. Using the more conservative Tukey's HSD pairwise test, the differences between successful pager use and diagnostic group disappeared but the sex difference remained, indicating that the pager was more effective for men than for women. However, examination of the results showed that this small difference was due entirely to the six participants who were significantly worse during the pager phase. When these six were excluded, none of the differences were significant. No differences to the results reported here were found when we excluded the exceptions to the randomisation procedure.

Preliminary analysis of the caregiver strain index indicates that the use of this paging system leads to a reduced stress in carers. These results will be reported separately.

\section{Discussion}

There is evidence that the paging system enabled most of our participants to carry out more everyday tasks than they were able to achieve without the pager. The results are not dissimilar to those from the pilot study, when a simpler A-B-A design (baseline, treatment, post-treatment baseline) was used. It should be remembered that the present study included people with a wider range of deficits and a wider age range than in our previous study. Indeed, many of the patients were referred to us in desperation by their therapists when all else had failed. Thus, if psychologists and therapists found a strategy that worked, they stuck with it. So our findings might have been even more impressive had we been more selective in our inclusion criteria. However, we wanted to demonstrate that the system was applicable to a wide range of people with mixed problems.

Not only were our inclusion criteria generous, we also found that the successful use was not confined to people of a particular age, sex, diagnostic group, level of impairment as measured by neuropsychological tests, time since insult, or from particular social circumstances. It is clear that this particular paging system is of benefit to a wide ranging group of people with brain injury with memory, planning, and organisational problems. Many participants needed minimal training with the pager before they could benefit. Others who were more impaired needed help from a carer over a longer period before coping independently. One person, for example, took almost 2 weeks to learn that the beeping sound came from his pager and a further 4 weeks to learn that he had to press the big button twice to get to the message. Once he had learnt this he started responding to the messages and achieving his goals.

Although the pager phase in the pilot study was 12 weeks, we have now shown that having the pager for only 7 weeks produces similar results. Many people who were referred because all else had failed did well here. What this paging system is able to do is to enable people with memory or planning problems to use the strategies taught by therapists by providing the consistent and reliable prompting that no family or carer can provide. What is more, not only can the system help implement errorless learning procedures, the paged reminders are not perceived as nagging because clients know that the only messages they receive are ones that they themselves have asked for. 
What about those who did not benefit from the pager? The earlier "dropouts" tended to be people who thought that they did not need the pager or those with poor insight. There is probably little point in pushing this system on people reluctant to use it. The other group who failed to complete the trial tended to include those participants whose circumstances had changed between the baseline phase and the later phases. For example, one man was moved from sheltered accommodation to his brother's farm where the targets monitored at baseline and prompted for the first 4 weeks of the pager phase were no longer relevant. One had a new girlfriend who reminded him to do things so he thought that he no longer needed the pager; other people left home or did not want to continue with the pager when they left hospital or rehabilitation. Of the six who deteriorated, one misunderstood the instructions for monitoring, two showed intellectual deterioration, a fourth developed psychiatric problems, a fifth had a carer who resigned during the monitoring period and there was no one to check that the forms were being filled in correctly, and the husband of the sixth tried to change the target behaviours and regular routines during the pager phase, leaving his wife totally confused.

The people for whom the paging system seems particularly useful are those with some (albeit limited) insight, sufficient vision to read the screen without too much effort, and a lifestyle in which it is helpful to carry out some tasks independently. For some of these people there is no doubt that the system can enhance independent living and improve morale by establishing new routines or building on systems already in place, often helping clients to achieve a level of independence thought no longer possible. For instance, one person was able to again use public transport independently, where previously he had been reliant on a carer.

In short, we have demonstrated in a randomised control trial with a crossover design, that this particular paging system significantly reduces everyday failures of memory and planning in people with brain injury at relatively low cost. Less than $50 \%$ of target behaviours were achieved without the pager, this rose to about $76 \%$ with the pager. Once the pager is removed, there seems to be some reduction in the target behaviours achieved (about $62 \%$ ) but this is still significantly more than in the baseline phase. For some persons 7 weeks with the pager was long enough to help establish routines, others may need the system on a longer term basis (this may partly be dependent on such factors as lifestyle, other systems already in place, and user confidence).

- The system used was NeuroPage ${ }^{2}$

- No differences from the results reported here were found when we excluded the exceptions to the randomisation procedure.

- The system is now offered by Lifespan NHS Trust at a cost of $£ 60$ per patient per month.

This work was supported by grants from NHS Executive Anglia and Oxford $\mathrm{R}$ and $\mathrm{D}$. We thank Larry Treadgold for the NeuroPage software, Philips for their gift of 50 pagers, Hutchinson for their cooperation in arranging air time, and Peter Watson for statistical advice.

1 Wilson BA, Watson PC. A practical framework for understanding compensatory behaviour in people with organic memory impairment. Memory 1996;4:465-86.

2 Hersh N, Treadgold L. NeuroPage: the rehabilitation of memory dysfunction by prosthetic memory and cueing. Nemorehabilitation 1994;4:187-97.

3 Wilson BA, Evans JJ, Emslie $\mathrm{H}$, et al. Evaluation of Nilson BA, Evans J, Emslie $\mathrm{H}$, et al. Evaluation of
NeuroPage: a new memory aid. $\mathcal{F}$ Neurol Neurosurg PsychiaNeuroPage: a new

try 1997;63:113-5.
4 Wilson BA, Emslie H, Quirk K, et al. George: learning to live independently with NeuroPage. Rehabil Psychol 1999;44: $284-96$.

5 Evans JJ, Emslie H, Wilson BA. External cueing systems in the rehabilitation of executive impairments of action. F Int Neuropsychol Soc 1998;4:399-408.

6 Baddeley AD, Emslie H, Nimmo-Smith I. The speed and capacity of language processing (SCOLP) test. Bury St Edmunds, Suffolk: Thames Valley Test Company, 1992.

7 Wilson BA, Cockburn J, Baddeley AD. The Rivermead behavioural memory test. Bury St Edmunds, Suffolk: Thames Valley Test Company, 1985.

8 Robertson IH, Ward T, Ridgeway V, et al. The test of everyday attention. Bury St Edmunds, Suffolk: Thames Valley Test Company, 1994.

9 Wilson BA, Alderman N, Burgess P, et al. Behavioural assessment of the dysexecutive syndrome. Bury St Edmunds, ment of the dysexecutive syndrome. Bury St

10 Robinson BC. Validation of a caregiver strain index. 7 Gerontol 1983;38:344-8.

1 Everitt B. Cambridge dictionary of statistics in the medical sciences. Cambridge: Cambridge University Press, 1995. 